\title{
Energy Efficient Algorithm for Wireless Sensor Network using Fuzzy C-Means Clustering
}

\author{
Abhilasha Jain \\ CSE Department \\ GZS Campus College of Engineering and Technology \\ Maharaja Ranjeet Singh, PTU, Punjab, India
}

\author{
Ashok Kumar Goel \\ ECE Department \\ GZS Campus College of Engineering and Technology \\ Maharaja Ranjeet Singh, PTU, Punjab, India
}

\begin{abstract}
Energy efficiency is a vital issue in wireless sensor networks. In this paper, an energy efficient routing algorithm has been proposed with an aim to enhance lifetime of network. In this paper, Fuzzy C-Means clustering has been used to form optimum number of static clusters. A concept of coherence is used to eliminate redundant data generation and transmission which avoids undue loss of energy. Intra-cluster and inter-cluster gateways are used to avoid nodes from transmitting data through long distances. A new strategy has been proposed to select robust nodes near sink for direct data transmissions. The proposed algorithm is compared with LEACH, MR-LEACH, MH-LEACH and OCM-FCM based upon lifetime, average energy consumption and throughput. From the results, it is confirmed that the performance of the proposed algorithm is much better than other algorithms and is more suitable for implementation in wireless sensor networks.
\end{abstract}

Keywords-WSN; clustering; sleep-awake; virtual grids; multihop; routing

\section{INTRODUCTION}

Wireless Sensor Networks (WSNs) consist of thousands of micro-sized low power Sensor Nodes (SNs) randomly deployed in the Sensor Field (SF). These SNs sense local environmental statistics, aggregate and communicate sensed information to sink. For each operation SN consumes its battery power. As SFs are hostile in most of the applications of WSN, it is not possible to replace batteries of SNs [1]. In order to enhance lifetime of network, the routing algorithms in WSN mainly focus on energy efficiency.

Clustering is one of the most effective techniques in routing to preserve the energy of the network. The whole network is organized into small groups of SNs called clusters. In each cluster, one node is elected as Cluster Head $(\mathrm{CH})$ which performs the task of collecting data from all Cluster Member (CMs) nodes, aggregation of data and forwarding it to the sink directly or in multi-hop manner [2], [3]. The aggregation may or may not be perfect depending upon the relation between the sensed data. Perfect aggregation means many k-bit data packets are compressed to a single k-bit packet. Perfect-fusion, a simple technique has attracted many researchers' interest [2], [4]. LEACH (Low Energy Adaptive Clustering Hierarchy) [2], EEUC (Energy Efficient Unequal Clustering) [3], IB-LEACH (Intra-Balanced LEACH) [5], MR-LEACH (Multi-hop Routing LEACH) [6], MH-LEACH (Multi-Hop LEACH) [7], EMRP (Energy efficient multi-hop routing protocol) [8], ACEEC [9], OCM-FCM (Optimal Clustering Mechanism Fuzzy-C Means) [10], SEECP (Stable
Energy Efficient Clustering Protocol) [11] and MLRC (MultiLevel Route-aware Clustering) [12] are some of the popular clustering protocols. In these protocols, all nodes in the network actively sense from the environment and continuously generate data. The nodes which are placed close to each other tend to generate redundant data due to phenomenon of coherence. A major amount of network energy is wasted in transmitting redundant data to sink which directly affects the network lifetime. Protocols like Span [13] and LEACH-SM (LEACH Spare Management) [14] keep a small subset of SNs active in such a way that these nodes cover the whole network while other nodes in the network are kept in sleep mode. In [15] an energy efficient sleep scheduling has been proposed in order to maintain network coverage and connectivity. Consequently, the energy consumed per round is reduced, thus, increasing the WSN lifetime.

Major part of energy is consumed by SNs in transmitting data which is proportional to the distance between sensor nodes raised to power $\beta(\beta \geq 2)$. Therefore, long-distance transmissions should be minimized for optimizing the usage of network energy [16]. Multi-hop data transmission between Gate Way nodes (GWs), CHs and sink can be used to reduce energy consumption, but SNs (may be CHs or GWs) near sink are more probable to be selected for transmitting the data to sink, resulting in their early death and thus, effecting the overall performance of the network.

In many clustering algorithms, number of clusters in each round is not fixed and employs poor $\mathrm{CH}$ election and cluster formation techniques. As a result, total inter and intra cluster distance becomes large, resulting in high energy consumption of the network. Therefore, soft computing techniques can be beneficially employed in cluster based routing protocols [16], [17]. Fuzzy-C means clustering groups the SNs based upon their degree of membership in each cluster. The aim is to minimize the sum of distances between the SNs and the centroid of their cluster. OCM-FCM uses Fuzzy-C means clustering to form more uniform and correlated clusters in order to improve the lifetime of WSN [10].

In this paper, a multi-hop efficient routing algorithm for WSN has been proposed. The algorithm emphasizes to optimize the energy usage in the network in order to enhance the network lifetime. It eliminates redundant data generation in the network by keeping only one node active from the set of nodes that are sending redundant data and also renders full network coverage. Initially, the whole network is divided into optimal number of static clusters using FCM. $\mathrm{CH}$ in each 
cluster changes deterministically during the network operation. In order to reduce long distance transmissions and balance load, multi-hop routes are established using gate way nodes. In multi-hop routing, the nodes which lie near the sink are more probable to be selected for establishing direct link to the sink despite of having lower residual energy. Therefore, SNs near the sink will die soon and create energy hole in the network. To avoid it, a strategy is designed which selects the nearest eligible SN for transmitting data to sink, therefore consuming least amount of energy. It also ensures that this SN has sufficient residual energy so that it may not become dead.

The remainder of the paper is organized as follows: Section 2 gives brief review of the related work. Network model and assumption made for the proposed work has been presented in Section 3. Section 4 describes the proposed algorithm for an energy efficient multi-hop adaptive routing using FCM clustering. The results and their comparison with other algorithms have been discussed in Section 5. Finally concluding remarks are given in Section 6.

\section{RELATED RESEARCH}

Many clustering protocols have been explored in literature for WSN in the last few years to increase network lifetime. LEACH is forerunner decentralized clustering protocol uses random rotation of $\mathrm{CH}$ in each round to evenly distribute the load in the network. CHs are responsible to transmit cluster information to the sink [2]. CHs far away from the sink consume lot of the energy to transmit data to sink. Randomized selection of $\mathrm{CHs}$ in each round adds extra overhead to the network. The algorithm does not assure even distribution of $\mathrm{CHs}$ in the network which may result into uneven energy consumption in different parts of the network. Moreover, the clusters formed in each round may or may not be correlated which increases the intra cluster distances. Therefore, LEACH is not suitable for large scale networks.

IB-LEACH is an improvement over LEACH [5]. CH selection of method is complex and involves lot of computations. Some nodes in the network are overburdened.

$\mathrm{MH}-\mathrm{LEACH}$ and MR-LEACH are the variants of LEACH, which follow the same $\mathrm{CH}$ selection methods as proposed in LEACH [6], [7]. These algorithms establish the multi-hop routes to transmit data to the sink and reduce number of long distance transmissions in the network. $\mathrm{CH}$ nodes which are near the sink (inner $\mathrm{CH}$ ) are responsible for transmitting data to the sink and other $\mathrm{CHs}$ (outer $\mathrm{CH}$ ) will transmit their data to the nearby $\mathrm{CH}$. When sink lies outside the sensor field, inner $\mathrm{CH}$ will consume higher energy than outer $\mathrm{CHs}$ and may die soon.

EEUC and EMRP divide the network into unequal sized clusters [3], [8]. The clusters that are formed near the sink are smaller in size as compared to clusters far away from the sink. There is an additional overhead involved for $\mathrm{CH}$ selection in each round. Nodes near sink are responsible to transmit data directly to sink even if they have low energy.

ACEEC and THCEEC [9] are the centralized routing algorithms which results in better network lifetime as compared to the conventional distributed routing protocols like LEACH. The algorithm divides the whole network in regions which act as static clusters for WSN. The sink finds $\mathrm{CH}$ in each region and efficient path to route region data to sink. This centralized scheme is helpful in planning power- aware, well-organized routes in the network. Network status needs to transmit to the sink in each round which increases energy consumption in all SNs and overall performance of network degrades.

Soft computing methods like Fuzzy C-Means clustering (FCM), K-means clustering, Genetic Algorithms (GA) and Particle Swarm Optimization (PSO) are recently used to overcome problems of non-correlated clusters [10], [17]. It helps to prolong the life of WSN. However, all these methods run at centralized node i.e. at sink and need physical location of SNs. In OCM-FCM reduces the cluster formation overhead on $\mathrm{SNs}$ as sink divides WSN in $\mathrm{K}$ optimal number of static clusters. After first round, the current $\mathrm{CH}$ selects a highest energy node in the cluster and elects it as $\mathrm{CH}$ for the next round. This algorithm uses multi-hop technique by finding Secondary Cluster Heads (SCHs) from already chosen $\mathrm{CHs}$ if sink is far away from the sensor field [18], [19]. It will result in reduction of long distance transmissions. The drawbacks of the algorithm are: 1) Poor $\mathrm{CH}$ selection; 2) $\mathrm{CHs}$ are overburdened as compared to other SNs; 3) SCHs consume more energy as compared to primary $\mathrm{CHs}$. In all the algorithms discussed, all nodes are regularly sensing and transmitting data to sink. Thus, large amount of network energy is wasted in redundant data transmissions which need to be reduced.

\section{NETWORK MODEL AND ASSUMPTIONS}

Total N sensor nodes in WSN are randomly deployed in of $\mathrm{X} \mathrm{m}$ by $\mathrm{Y} \mathrm{m}$ rectangular sensor field. The assumptions made for the implementation of the proposed work are as given under:

1) All nodes are stationary and have unique ID.

2) It is assumed that all SNs find their locations either through GPS system or using some localization algorithm.

3) All nodes are static and have same initial energy $E_{\text {init }}$

4) All the active sensor nodes regularly generate information from the environment.

5) All $\mathrm{SNs}$ are capable to perform data aggregations in perfect fusion mode.

6) In the cluster formation process, each $\mathrm{SN}$ can be a member of only one cluster and can act as $\mathrm{CH}$ for the same cluster.

7) The sink has unlimited energy and computational resources.

8) First order radio energy model described in [2] is utilized for sensor nodes during their communications procedure.

\section{PROPOSED ENERGY EFFICIENT AlgORITHM}

The paper proposes an energy efficient algorithm for WSN using multi-hop routing. Initially it runs a network dimensioning phase where sink divides the whole network into desired number of static clusters using FCM technique and turns off all SNs producing redundant data. Thereafter, it organizes the network operation into a series of rounds where each round is divided into network setup phase and network communication phase. In network set up phase, new $\mathrm{CHs}$ are elected in static clusters if required and energy efficient multihop paths are established from each SN to the sink. The network communication phase deals with intra-cluster and 
inter-cluster multi-hop routing and data transmissions. In network communication phase every $\mathrm{CH}$ allocates TDMA slots to $\mathrm{CMs}$ in each cluster and for inter-cluster communication is achieved through CDMA codes.

\section{A. Network Dimensioning Phase}

a) Division of SF in granules: WSN is initially divided uniformly into granules. The size of granule may vary according to degree of coherence in sensed data. In a SF, if information to be sensed is more coherent, then size of granule may be taken large and vice-versa. The entire SF is divided into $n_{g}$ granules where $n_{x}$ granules are along $\mathrm{x}$-axis and $n_{y}$ granules along y-axis. The size of granule is $x_{w}$ by $y_{w}$ subject to constraint that $x_{w}$ and $y_{w}$ are integer multiple of width and height of SF respectively. Any $i^{\text {th }} \mathrm{SN}$ with co-ordinates $\left(x_{i}, y_{i}\right)$ finds its associated granule ID using formula given as under:

$$
\text { GranID }=\operatorname{round}\left(\frac{x_{i}}{x_{w}}+0.5\right)+\left(\operatorname{round}\left(\frac{y_{i}}{y_{w}}+0.5\right)-1\right) \times y_{n}
$$

All SNs save this information for further use.

b) Initial Active-Sleep Methodology: Once SNs find their associated granule ID, each SN will broadcast a message $S$ (NodeID, GranID) to all SNs in its radius $R_{\text {com }}$. Where $R_{c o m}$ is equal to the diagonal of a granule and is given by following equation:

$$
R_{\text {com }}=\sqrt{x_{w}^{2}+y_{w}^{2}}
$$

Initially, SNs do not need to broadcast their residual energy as all SNs have same $E_{\text {init }}$. Each SN saves NodeID of the received message if it belongs to its granule node and discards otherwise. In this way, all SNs maintains NodeID and $E_{\text {resi }}$ of its granule SNs. Once, all SNs save information of their associated GranID, granule SNs. SN with highest node ID in a granule will activate itself and broadcast its location and ID information to the sink. Remaining nodes in the granule shall be in sleep mode.

c) Clusters Formation: At the end of active-sleep process, the sink will receive location information of all $n$ active nodes. The sink first calculates their associated GranIDs using (1), then divides whole WSN into $K$ optimal number of clusters using FCM clustering algorithm. The SN is associated with the cluster in which it has the highest membership value based upon its distance from the centroids of the clusters. The sink broadcasts messages $S($ ClusterID,$<$ List of associated GranIDs $>)$ for each cluster to all SNs. SNs belonging to GranID specified in the message will save this message in its cluster table.

d) Initial Selection of Cluster Heads: The sink divides the whole network into $K$ clusters. Thereafter, the sink evaluates the fitness of all SNs in each cluster on the basis of an objective function given by (3). The SN with highest fitness value will be elected as $\mathrm{CH}$ in each cluster. The sink broadcasts this information to each cluster.

$$
f(i)=\sum_{j} \frac{1}{\operatorname{dis}(i, j)^{\beta}}, \forall j \in c^{\text {th }} \text { cluster }, j \neq i, 1 \leq c \leq K
$$

where $f(i)$ is the fitness of $i^{\text {th }}$ member of $c^{\text {th }}$ cluster. $\operatorname{dis}(i, j)$ is the Euclidean distance between $i^{\text {th }}$ and $j^{\text {th }}$ member of $c^{\text {th }}$ cluster. The value of $\beta$ is taken as 2 for $\operatorname{dis}(i, j)$ less than threshold distance, otherwise 4 .

e) Logical Region Division: In many multi-hop routing algorithms proposed by various researchers, SNs nearer to sink carry heavier traffic loads and are responsible for routing data to the sink. Therefore, SNs around sink deplete their energy at faster rate and may create energy hole near the sink. To avoid the problem, the SF under consideration is divided into three logical regions as Low Energy Area (LEA), Medium Energy Area (MEA) and High Energy Area (HEA) depending upon the energy required by SNs in these regions to transmit data directly to the sink. The sink assigns region IDs to all SNs. The logical region division has been shown in Fig. 1. The SNs from the region specified by the sink will only establish direct communication to it. Initially, LEA becomes region of interest.

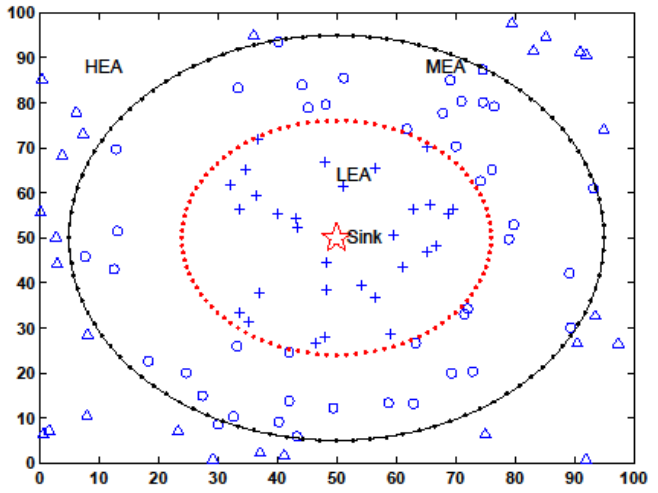

Fig. 1. Logical Region Division of SF in LEA, MEA and HEA (this figure is created for this research work).

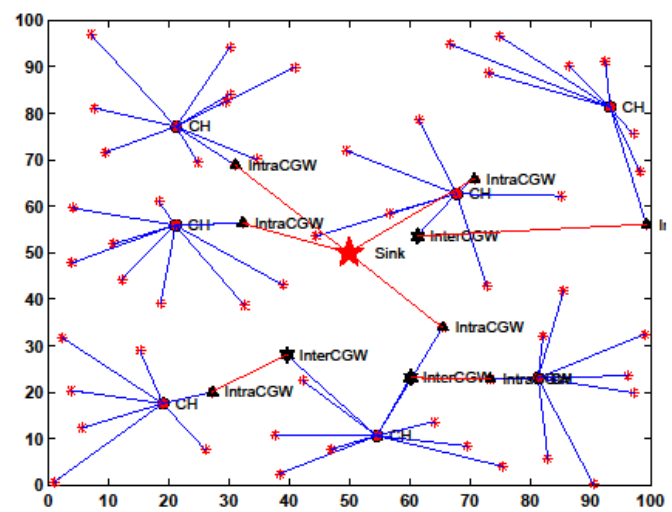

Fig. 2. Multi-hop topology for proposed system (this figure is created for this research work). 


\section{B. Network Setup Phase}

After initial set up of WSN by sink, multi-hop energy efficient routes are established for each $\mathrm{SN}$ in distributive manner. Re-election of CHs may start from next round at each cluster level.

a) $\mathrm{CH}$ Election: A $\mathrm{CH}$ of a cluster continues to be $\mathrm{CH}$ for the next rounds provided its residual energy $E_{\text {resi }}$ is greater than threshold energy of its cluster, $E_{T h}^{C}$. The technique eliminates the overhead of forming $\mathrm{CHs}$ in each round and saves energy. For its implementation, threshold energy of each cluster $E_{T h}^{C}$ begins with $60 \%$ of $E_{\text {init }}$ and decreased by $20 \%$ when energy of all nodes in the cluster falls below the threshold level. When $E_{\text {resi }}$ of $\mathrm{CH}$ goes below $E_{T h}^{C}$, it prompts new $\mathrm{CH}$ election process and activates the active-sleep process in the cluster. Each active $\mathrm{SN}$ in the cluster scans its granule to finds a SN (in sleep mode) with highest $E_{\text {resi }}$. Make specified SN active and goes itself in sleep mode. Before going to sleep mode it transmits all its routing information to newly selected active node. Then, present $\mathrm{CH}$ finds candidate $\mathrm{CH}$ nodes, having residual energy above $E_{T h}^{C}$. Candidate $\mathrm{CH}$ with maximum fitness calculated according to (3) is selected as new $\mathrm{CH}$. If there are two or more nodes with same fitness value, then, a node with higher ID number is chosen.

b) Multi-hop route establishment: $\mathrm{CH}$ in a cluster receives information from its $\mathrm{CMs}$, aggregates the received data with its own sensed data and forwards it to sink either in single hop or in multi-hop. CMs are accountable to sense environmental data and transmit sensed information to $\mathrm{CH}$. Therefore, $\mathrm{CH}$ in a cluster does more work than other nodes and consumes much higher energy. To reduce its work, the task of inter cluster communication is shifted to another node known as Intra Cluster GW (IntraCGW). The cluster $\mathrm{CH}$ will choose IntraCGW in the direction of logical region announced by sink. If the selected IntraCGW is in the announced logical region, link is established directly to sink depending upon a probability function described in (4), otherwise inter cluster path is devised. Then, IntraCGW finds a nearest inter cluster $\mathrm{SN}$ in a cluster, currently not connected in order to avoid loops in communication path. The selected inter cluster node called "Inter Cluster GW (InterGW)" forms the link between the two clusters. The multi-hop topology formed by the proposed algorithm is shown in Fig. 2.

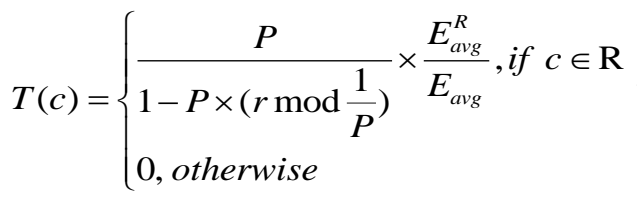

where $\mathrm{P}$ is the desired percentage of direct links to the sink, $\mathrm{r}$ is the current round, $E_{\text {avg }}^{R}$ is the average energy of CMs of the cluster in announced region $R, E_{\text {avg }}$ is the average energy of the active nodes in the network. Each $\mathrm{CH}$ in $c^{\text {th }}$ cluster in announced region generates a random number between 0 and 1 . The $\mathrm{CH}$ will establish a link to the sink through selected IntraGW, if generated random number is less than threshold value of the cluster as calculated in (4). The proposed algorithm to establish multi-hop routes is given in Fig. 3.

c) Region Announcement by Sink: In order to attain nearly balanced energy depletion and to increase the reliability of WSN, it is desirable that all nodes remain alive for larger time period. SNs in LEA region consume lowest energy in direct data transmissions than MEA and HEA region nodes. Therefore, nodes from LEA region are desirable for establishing direct link to sink, but if these nodes continue to transmit information directly to sink, they will consume energy continuously and ultimately become dead. To avoid such situation, a shifting strategy between three logical regions has been proposed in this paper. A threshold parameter $E_{t h}^{R}$ is taken to decide this shifting strategy. Initially, $E_{t h}^{R}$ is set to $30 \%$ of $E_{\text {init }}$ energy. Depending upon its value, the sink announces the region of interest in each round. If residual energy of all SNs in the SF goes below $E_{t h}^{R}$ then $E_{t h}^{R}$ is decreased to $10 \%$ of $E_{\text {init }}$ and announce LEA as region of interest.

1. For each $\mathrm{CH}$ check $\left(E_{\text {resi }}^{\mathrm{CH}}<E_{T h}^{C}\right)$

2. Broadcast a query "What is the highest residual energy of the granule?" to all CMs and activate active sleep process.

3. Each active $\mathrm{SN}$ in the cluster finds other $\mathrm{SN}$ in its granule with highest $E_{r e s i}$. Activate newly selected SN. Transfer its routing information and goes in sleep mode.

4. All the activated nodes send reply message to $S$ (NodeID, GranID, $\left.E_{\text {resi }}\right)$ current $\mathrm{CH}$.

5. Current $\mathrm{CH}$ finds a set $\mathrm{CCH}$ of candidate $\mathrm{CHs}$ such that their $\left(E_{r e s i} \geq E_{T h}^{C}\right)$

6. Check if no $\mathrm{CCH}$ is selected $E_{\text {resi }}^{C H}$ is decreased by $20 \%$ and current $\mathrm{CH}$ remains $\mathrm{CH}$ for next the rounds. Otherwise

Select a candidate $\mathrm{CH}$ with maximum fitness value and broadcast it to all SNs in the cluster.

7. Multi-Hop Route Establishment to Sink by Selected CH

a. $\mathrm{CH}$ chooses $\mathrm{GW}$ within its cluster in the direction of announced region of interest with highest $E_{\text {resi }}$.

b. Check selected GW is in Region of Interest and $\left(E_{r e s i} \geq E_{t h}^{R}\right)$

$\mathrm{CH}$ generates a random number $<1$; if it is less than threshold of its cluster then establish connection to the sink.

Otherwise

Check if all other clusters in the network are connected directly or indirectly to it. Establish connection to the sink

Otherwise $G W$ selects another closest SN from other clusters not connected to it and

Establish connection to it.

\section{Network Communication Phase}

In communication phase, there are two types of communication takes place in the network: intra-cluster 
communication and inter cluster communication. For intra cluster communication $\mathrm{CH}$ node assigns TDMA schedule to its entire CMs. Each CM in a cluster transmits its sensed data to $\mathrm{CH}$ in its allotted slot. With TDMA scheme lot of energy of CMs is saved as CM goes in sleep mode when it is not transmitting any data. $\mathrm{CH}$ of each cluster receives data packets from $\mathrm{CMs}$ and aggregated the received information with its own sensed data and forwards it to IntraGW. It will then aggregate the received data packet with its own and forward it to Inter $G W$ using CDMA codes. In each round, active SN those go below $E_{t h}^{R}$ transmit its energy status to the sink. Depending upon information received, it announces the region of interest.

\section{RESULTS AND DISCUSSIONS}

The proposed methodology has been implemented with MATLAB and executed on 100 randomly deployed wireless sensor networks in order to evaluate the performance parameters: network lifetime, energy consumption and throughput. Network lifetime is considered as the number of rounds until the complete network is dead. Energy consumption in each $\mathrm{SN}$ of the network is measured as the sum of energy consumed in receiving, aggregating and forwarding the data to other intermediate nodes or to the sink. Throughput is defined as sum of unique data packets generated in each round. For example, four data packets are generated from an active granule by four SNs in that granule. We consider it as one because other three carries same data as that of first packet. For simplicity, it is assumed that there is no data collision and packet loss in the wireless channel. Likewise, control packets have also been ignored for the evaluation of performance parameters.

In this simulation setup, one hundred static sensor nodes are randomly distributed in a SF of area 200m X 200m and considered as one WSN deployment. The sink is placed at location $(100,100)$ and is immobile. Initial battery power for all $\mathrm{SN}$ is taken as $0.5 \mathrm{~J}$. Further, to ensure the generalisation of results, the simulation experiments were conducted for one hundred WSN deployments. Each simulation is run until all sensor nodes in the network become dead. The energy consumed in the network is calculated for transmitting, receiving and aggregating data packets. The energy consumed in aggregating and transmitting 1 bit data over distance $d$ is calculated using (5).

$$
e_{t}=\left\{\begin{array}{l}
E_{\text {elec }}+E_{D A}+E_{f s} d^{2}, \forall d \leq d_{o} \\
E_{\text {elec }}+E_{D A}+E_{m p} d^{2}, \forall d>d_{o}
\end{array}\right.
$$

Energy consumed in receiving 1 bit data is given as under:

$$
e_{r}=E_{\text {elec }}
$$

where $E_{\text {elec }}$ is energy dissipated per bit to run transmitter or receiver circuit, taken as $50 \mathrm{~nJ} / \mathrm{bit} . E_{D A}$ is energy consumed in aggregating one bit data and set to $5 \mathrm{~nJ} / \mathrm{bit} / \mathrm{signal} . E_{f s}$ and $E_{m p}$ are energy consumed in free space model and energy used in multipath fading model taken as $10 \mathrm{pJ} / \mathrm{bit} / \mathrm{m}^{2}$ and $0.0013 \mathrm{pJ} / \mathrm{bit} / \mathrm{m}^{4}$ respectively. The threshold distance $d_{o}$ is equal to square root of ratio $\frac{E_{f s}}{E_{m p}}$. Total energy consumed in communication from $S N_{i}$ to $S N_{j}$ over distance $d$ is given by (7).

$$
E_{i j}(l, d)=l *\left(e_{t}+e_{r}\right)
$$

Where $l$ is the length of data packet taken as 4000 bits. To decide optimal number of clusters $K_{\text {opt }}$ for the proposed algorithm, the algorithm is executed on all 100 simulations for varying $K$, i.e. number of clusters, from 1 to 20 . The average energy consumption per round for each value of $K$ is calculated. The minimum average energy consumption per round is found at $K=10$. Hence, the value of $K_{\text {opt }}$ is taken as10.

In order to generalize the results, all the five algorithms (LEACH, MR-LEACH, MH-LEACH, OCM-FCM and EEAFCM) were implemented on the same hundred deployments and averaged results obtained from them have been used to present the comparative account of the algorithms. The results are shown in Fig. 4, 5, 6, 7 and 8.

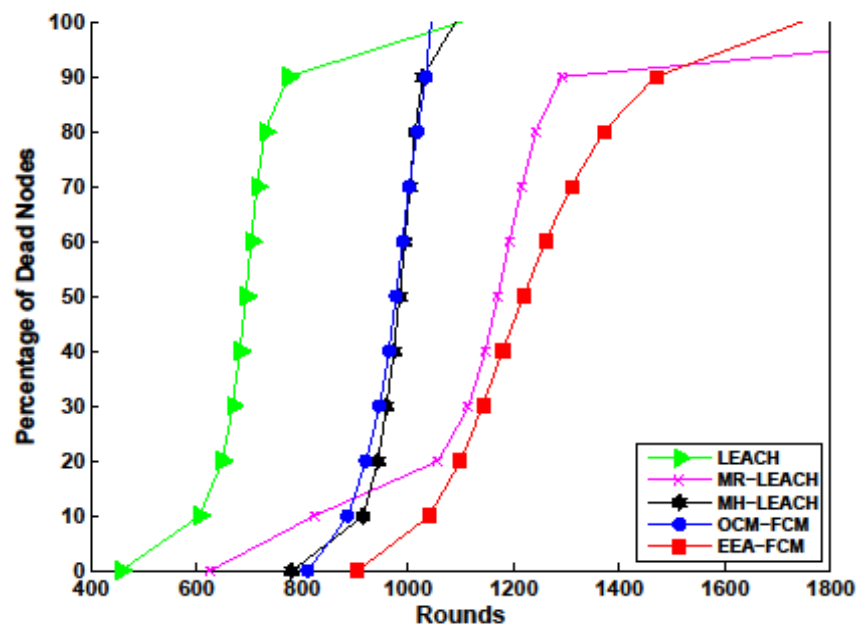

Fig. 3. Graph of number of operational nodes verses rounds in WSN for $\mathrm{N}=100$.

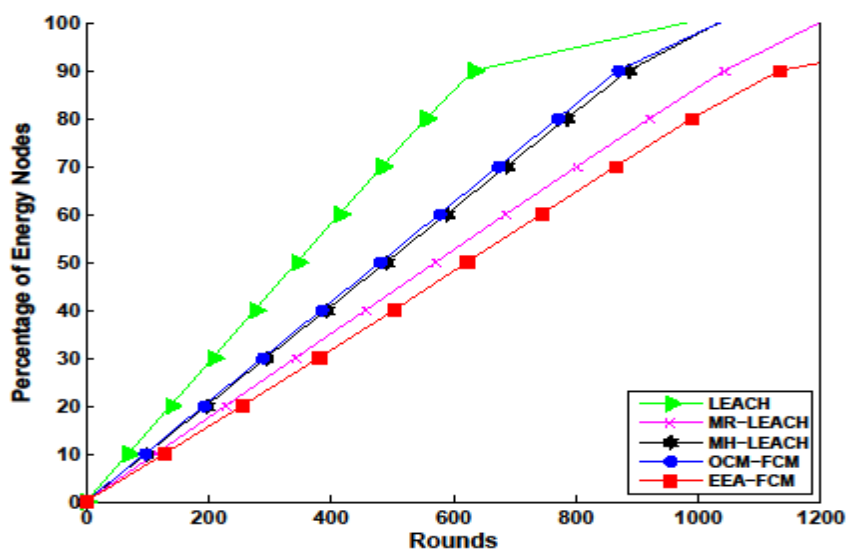

Fig. 4. Energy consumption in WSN for $\mathrm{N}=100$. 


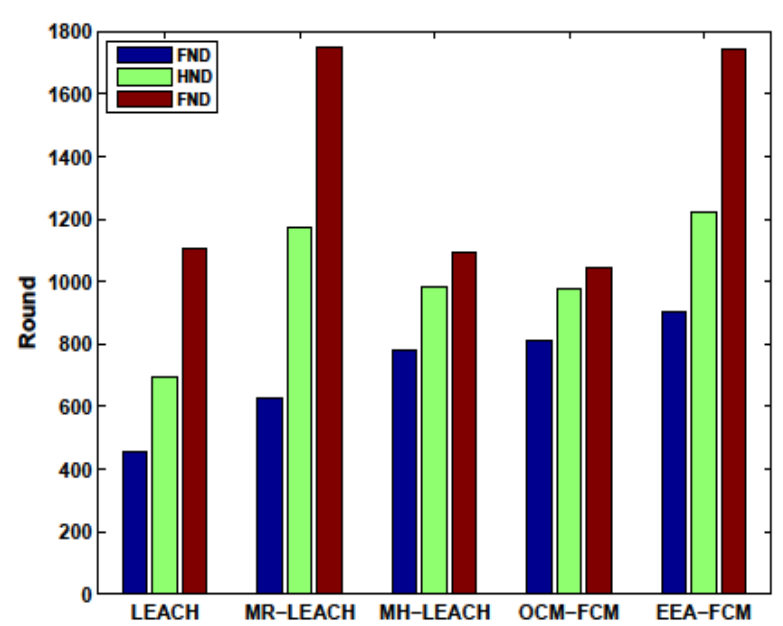

Fig. 5. FND, HND and LND for WSN for $\mathrm{N}=100$.

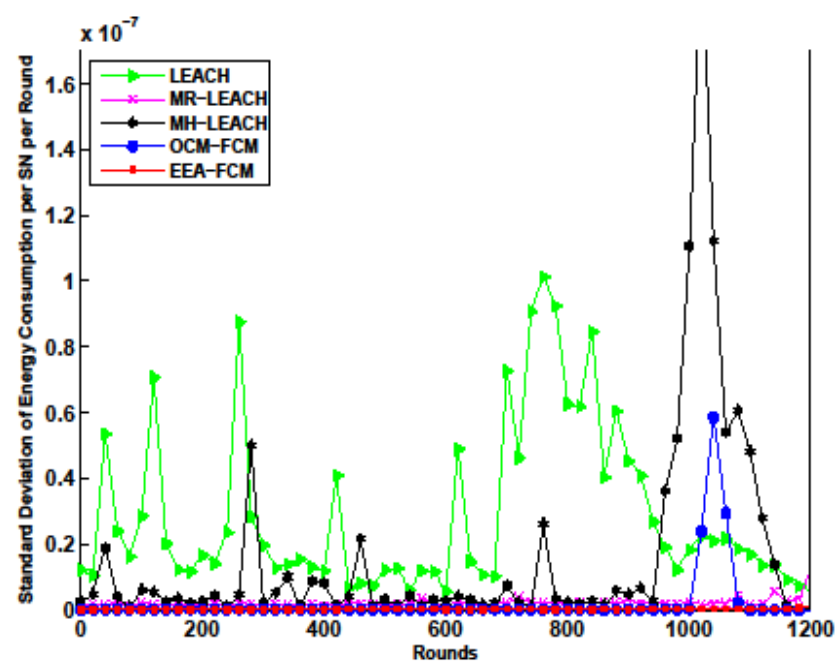

Fig. 6. Standard deviation of average energy consumption per SN per Round in WSN for $\mathrm{N}=100$.

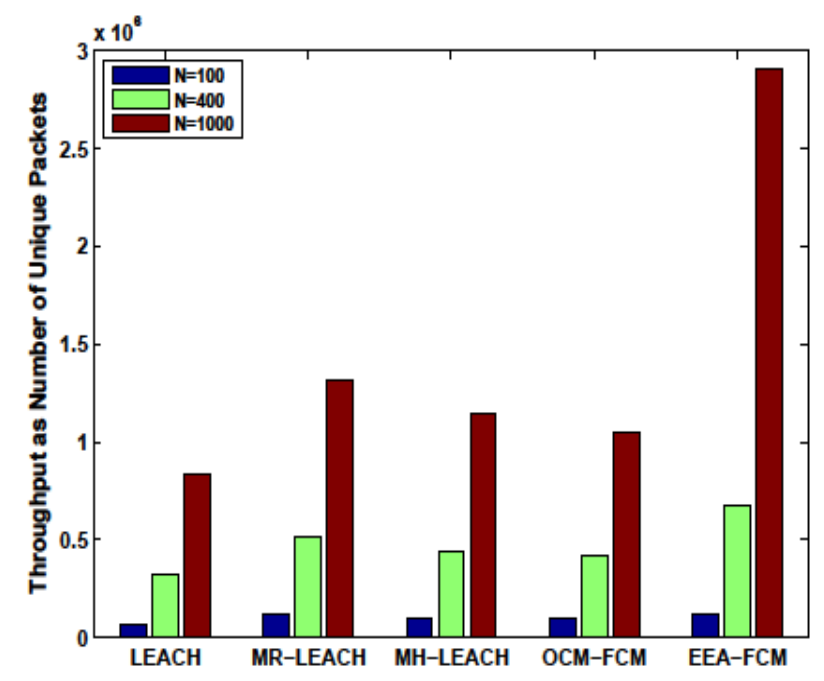

Fig. 7. Comparative plot of throughput for $\mathrm{N}=100,400$ and 1000.
The graph of number of operational nodes verses the number of rounds is shown in Fig. 4. It is clearly observed from the figure that EEA-FCM remains operational for longer period of time as compared to LEACH, MR-LEACH, MHLEACH and OCM-FCM algorithms. At $900^{\text {th }}$ round, the dead node percentage increases to 7, 10 and 14 for $\mathrm{MH}$-LEACH, MR-LEACH and OCM-FCM algorithms respectively while it is $1 \%$ for EEA-FCM. Similarly, when EEA-FCM lost its $10 \%$ network operational ability (i.e. $10 \%$ nodes are dead), network in OCM-FCM and MH-LEACH almost ceases to exist. First $10 \%$ nodes in MR-LEACH died more quickly as compared to MH-LEACH, OCM-FCM and EEA-FCM. Network in MRLEACH stabilizes to some extent after losing $20 \%$ of its operational ability. This shows EEA-FCM algorithm performs far better than the other algorithms under test at each point of network operation.

Percentage of total energy consumed in all the five protocols has been presented in Fig. 5. It is observed from the figure that the rate of energy consumption in $\mathrm{LEACH}$ is much higher as compared to EEA-FCM, OCM-FCM, MR-LEACH and MH-LEACH. Energy consumption in OCM-FCM and $\mathrm{MH}-\mathrm{LEACH}$ is almost same. It is worth noting that OCMFCM is transmitting cluster data to the sink in single hop whereas MH-LEACH transmits in multiple hops. Therefore, OCM-FCM clustering technique is much better as compared to MH-LEACH. At the point of network operation where EEA-FCM consumed fifty percent of its network energy, OCM-FCM and MR-LEACH consumed $65 \%$ and $60 \%$ respectively. Hence, energy usage of the network is more economical in EEA-FCM as compared to OCM-FCM and MR-LEACH.

From Fig. 4 and 5, it is clearly observable that distribution of network load amongst all the SNs is more balanced which will enhance the network lifetime. Round value for FND (First Node Dead), HND (Half Node Dead) and LND (Last Node Dead) has great significance to evaluate the performance of network. The round value of FND indicates the reliability and stability of the network operation. The round value of HND specifies rate of decay of performance of network after first node become dead. The round value for LND indicates the duration for which the network remains operational. Fig. 6 shows that FND, HND and LND for EEA-FCM is much higher as compared to compared to OCM-FCM, MH-LEACH, MR-LEACH and LEACH algorithms. In the proposed EEAFCM algorithm, data collection, aggregation and data forwarding is divided amongst the $\mathrm{CH}$ and $\mathrm{GW}$ nodes of the cluster which results in healthier $\mathrm{CH}$ node for long period of time. Due to these factors, energy usage in the network and decay in reliability of WSN operation is reduced.

The standard deviation of node's average consumption per SN per round is shown in Fig. 7. Standard deviation value of average energy consumption in a $\mathrm{SN}$ at any round indicates load distribution amongst all SNs in the network. Large value of standard deviation means that there is large fluctuation from mean of average energy consumption in a $\mathrm{SN}$. It indicates that some nodes in the network consume large amount of energy as compared to other nodes. OCM-FCM shows higher value of standard deviation, when less number of nodes in the network are alive. It is worth noting the standard deviation of energy consumption per SN in EEA is very small as compared to LEACH, MR-LEACH, MHLEACH and OCM-FCM. 


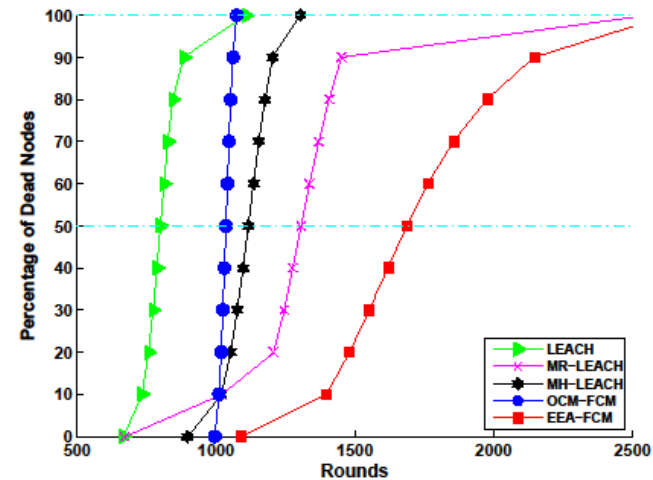

Fig. 8. Percentage of dead nodes verses rounds at number of nodes is 400 .

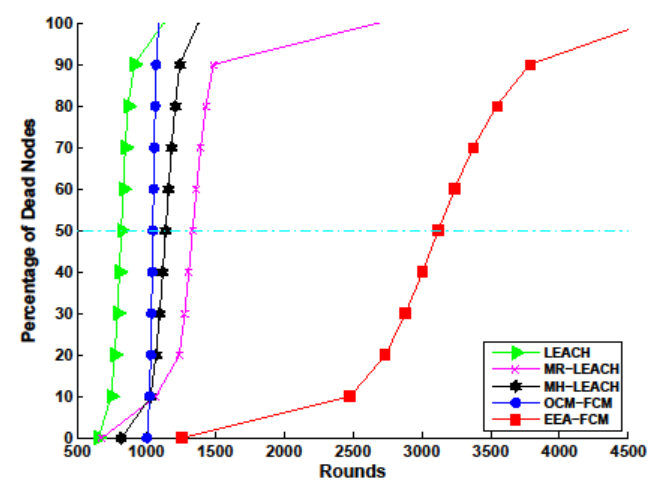

Fig. 9. Percentage of dead nodes verses rounds at number of nodes is 1000.

Therefore, network load is uniformly distributed amongst all SNs in EEA-FCM and consume almost equal amount of energy which leads to more stable and reliable network operation.

A comparative graph of throughput for all five algorithms is presented in Fig. 8. OCM-FCM and MR-LEACH acquire less amount data from the SF as their nodes have lesser lifetime as compared to EEA-FCM. In EEA-FCM, SNs are not generating redundant data from SF and remain alive for longer period. Therefore, throughput of the network is improved. Total throughput in case of EAA-FCM is $7.6 \%$ higher than MR-LEACH and 28\% more than OCM-FCM. Thence, the proposed algorithm shows a significant improvement in throughput.

Node density of WSN may greatly affect SF coverage, network connectivity and network life time. Therefore, it is an important factor that needs to be considered while evaluating the performance of WSN. To verify the effect of node density on the performance of the network, additional simulation experiments are conducted by varying the number of nodes, $\mathrm{N}$, in the WSN deployments as $\mathrm{N}=100,400$ and 1000. Please note that $\mathrm{N}=400$ in $\mathrm{SF}$ of $200 \mathrm{~m} \mathrm{X} \mathrm{200m} \mathrm{is} \mathrm{equivalent} 100$ nodes in an area of $100 \mathrm{~m} \times 100 \mathrm{~m}$.

The percentage of dead nodes for varying number of rounds has been shown in Fig. $4(\mathrm{~N}=100)$ and Fig. $10(\mathrm{~N}=400)$ and Fig. $11(\mathrm{~N}=1000)$. Comparison for all three algorithm for $\mathrm{N}=100$ has already been discussed earlier. Taking the discussion further, it is seen that when network node density increased to 400, within first 1100 rounds, all nodes for LEACH, MH-LEACH and OCM-FCM become dead, but all the four hundred nodes for EEA-FCM are still alive. When $15 \%$ nodes of EEA-FCM are dead, $90 \%$ nodes in MR-LEACH has already become dead. Further, the slops of five curves in Fig. 6 have been observed. It is clearly seen that the slop of curve for EEA-FCM is much less than other two curves. This indicates that the rate of nodes becoming dead is much slower in EEA-FCM. Lifetime of WSN for the proposed algorithm is almost double than LEACH, MH-LEACH and OCM-FCM algorithms. Further, when number of nodes in WSN increased from 400 to 1000 , similar behaviour of algorithms has been observed. There is no improvement in network lifetime of LEACH, MH-LEACH, MR-LEACH and OCM-FCM. Network lifetime of EEA-FCM is far improved over other two algorithms. It is approximately 4.5 times that of $\mathrm{LEACH}, \mathrm{MH}-$ LEACH and OCM-FCM.

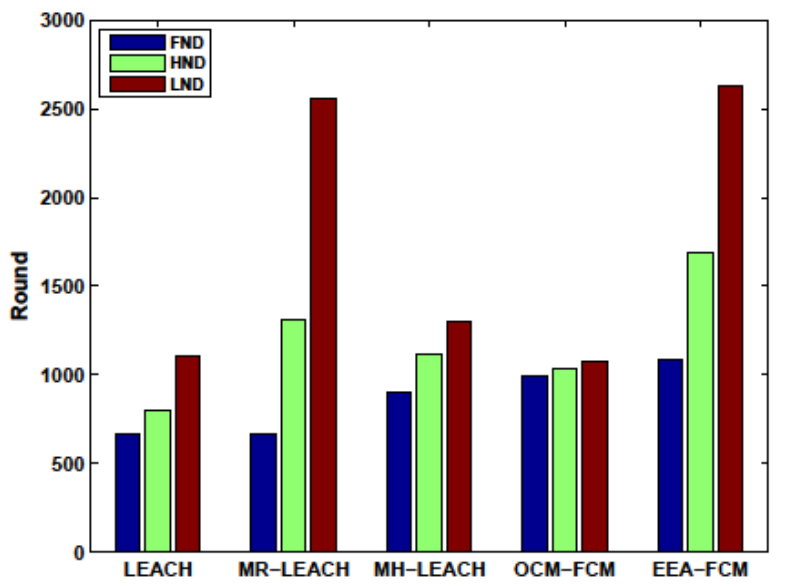

Fig. 10. FND, HND and LND for WSN for Varying Number of Nodes is 400.

The network throughput obtained in LEACH, MRLEACH, MH-LEACH, OCM-FCM and EEA-FCM with varying $\mathrm{N}$ is shown in Fig. 9 and Table $\mathrm{I}$. It is observed, for $\mathrm{N}=100$, OCM-FCM, MR-LEACH and EEA-FCM generate 96641,114973 and 123577 data packets in its lifetime, respectively. There is a $63 \%$ improvement in throughput of EEA-FCM over OCM-FCM for $\mathrm{N}=400$. By varying nodes from 400 to 1000, throughput obtained in EEA-FCM is almost three times as compared to OCM-FCM. The throughput of EEA-FCM is improved in many folds compare to LEACH and OCM-FCM with the increase in node density.

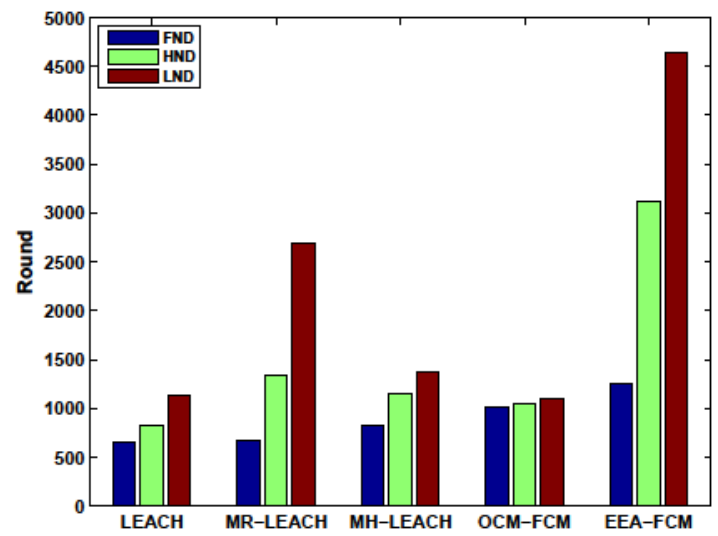

Fig. 11. FND, HND and LND for WSN for Varying Number of Nodes is 1000 
The value of rounds for First Node Dead (FND), Half Node Dead (HND) and Last Node Dead (LND) for all algorithms under examination at $\mathrm{N}=100,400$ and 1000 are shown in Fig. 10 and 11. For N=100, OCM-FCM improves the round number for FND by $4 \%$ over MH-LEACH. EEAFCM improves FND of MH-LEACH further by another $11 \%$. The round number for HND is $694^{\text {th }}, 1171^{\text {th }}, 984^{\text {th }}, 977^{\text {th }}$ and $1220^{\text {th }}$ round in LEACH, MR-LEACH, MH-LEACH, OCMFCM and EEA-FCM respectively.MR-LEACH improves the round number for HND for OCM-FCM by $19 \%$. EEA-FCM improves it further by $5 \%$. The LND round number is almost same for LEACH and OCM-FCM. The network lifetime for
EEA-FCM is improved 58\% over LEACH. It is also observed that by changing $\mathrm{N}$ from 100 to 400 , FND values of all three algorithms are improved. EEA-FCM improves HND value of OCM-FCM by $63 \%$. Network lifetime for EEA-FCM is almost double as compared to the other two algorithms. For $\mathrm{N}=1000$, there is not much improvement in LEACH and OCM-FCM. The round number for FND, HND and LND in EEA-FCM is delayed by 182, 1396 and 2063 rounds respectively as compared to their values at $\mathrm{N}=400$. Therefore, network operation got improved in EEA-FCM on increasing the node density.

TABLE I. LIFETIME AND ThroughPut For VARYING NoDE DENSITY N=100, 400 AND 1000

\begin{tabular}{|l|l|l|l|l|l|l|}
\hline Algorithm & Lifetime & Throughput & Lifetime & Throughput & Lifetime & Throughput \\
\hline & $\begin{array}{l}\text { Node Density } \\
\text { N=100 }\end{array}$ & $\begin{array}{l}\text { Node Density } \\
\text { N=400 }\end{array}$ & $\begin{array}{l}\text { Node Density } \\
\text { N=1000 }\end{array}$ \\
\hline LEACH & 1102 & 70093 & 1109 & 323570 & 1129 & 829786 \\
\hline MR-LEACH & 2375 & 114973 & 2559 & 512385 & 2684 & 1311447 \\
\hline MH-LEACH & 1092 & 97638 & 1305 & 445357 & 1381 & 1139135 \\
\hline OCM-FCM & 1044 & 96641 & 1075 & 414114 & 1093 & 1048670 \\
\hline EAA-FCM & 1746 & 123577 & 2634 & $\begin{array}{l}677378 \\
\text { in Wireless Sensor Networks. In proceedings of The Thirteenth }\end{array}$ \\
\hline
\end{tabular}

\section{CONCLUSION}

In this paper, an energy efficient algorithm for WSN is presented. The results have been compared with LEACH, MH-LEACH, MR-LEACH and OCM-FCM algorithms. From the outcome, it is concluded that lifetime and throughput of proposed algorithm is $58 \%$ and $28 \%$ more as compared to the other algorithms under examination. Network load amongst all SNs is optimally distributed in the proposed algorithm. The rate of decay i.e. the nodes becoming dead is also minimum for the proposed algorithm as compared to other algorithms. Therefore, it is concluded that EEA-FCM achieves better energy efficiency and enhanced lifetime. Moreover, the performance of network has also been analyzed for varying $\mathrm{N}=100,400$ and 1000. The performance of EEA-FCM is far better for higher node density of WSN. From the results, it is concluded that the proposed EEA-FCM algorithm out performs other algorithms discussed in this paper.

\section{REFERENCES}

[1] Akyildiz, F., Su, W., Subramaniam, Y. S., \& Cayirci, E. (2002).Wireless sensor networks: a survey. Computer Networks, 38(4), 393-422.

[2] Heinzelman, W. R., Chandrakasan, A., \& Balakrishnan, H. (2000). Energy efficient communication protocol for wireless microsensor networks. In proceedings of 33rd annual Hawaii international conference on System Sciences, IEEE, http://doi.org/10.1109/HICSS.2000.926982.

[3] Chen, G., Li, C., Ye, M., \& Wu, J. (2009). An unequal cluster-based routing protocol in wireless sensor networks. Wireless Networks, 15(2), 193-207.

[4] Shin, J., \& Suh, C. (2011). CREEC: Chain routing with even energy consumption. Journal of Communications and Networks, 13(1), 17-25.

[5] Salim, A., Osamy, W., \& Khedr, A. M. (2014). IBLEACH: Intrabalanced LEACH protocol for wireless sensor networks. Wireless Networks, 20(6), 1515-1525.

[6] Farooq, M. O., Dogar, A.B., \& Shah, G.A. (2010). MRLEACH: Multihop Routing with Low Energy Adaptive Clustering Hierarchy. In proceedings of fourth international conference on Sensor Technologies and Applications (SENSORCOMM), (pp. 262 - 268).

[7] Neto, J. H. B., Rego, A. S., Cardoso, A. R., \& Celestino Jr., J. (2014). MH-LEACH: A Distributed Algorithm for Multi-Hop Communication
[8] Huang, J., Hong, Y., Zhao, Z., \& Yuan, Y. (2017). An energy-efficient multi-hop routing protocol based on grid clustering for wireless sensor networks. Cluster Computing, 20(4), 3071-3083.

[9] Aslam, M., Munir, E. U., Rafique, M. M., \& Hu, X. (2016). Adaptive energy-efficient clustering path planning routing protocols for heterogeneous wireless sensor networks. Sustainable Computing Informatics \& Systems, $12, \quad 57-71$, http://dx.doi.org/10.1016/j.suscom.2017.08.001

[10] Su, S., \& Zhao, S. (2017). An optimal clustering mechanism based on Fuzzy-C means for wireless sensor networks. Sustainable Computing Informatics \& Systems, http://dx.doi.org/10.1016/j.suscom.2017.08.001

[11] Mittal, N., Singh, U., \& Sohi, B. S. (2016) "A stable energy efficient clustering protocol for wireless sensor networks," Wireless Networks, pp. 1-13, 2016.

[12] Sabet, M. \& Naji, H. (2016). An energy efficient multi-level route-aware clustering algorithm for wireless sensor networks: A self-organized approach. Computers \& Electrical Engineering, 56, 399-417.

[13] Chen, B., Jamieson, K., Balakrishnan, H., \& Morris, R. (2002). Span: An energy-efficient coordination algorithm for topology maintenance in ad hoc wireless networks. Wireless Networks, 8, 481-494.

[14] Bakr, B. A., \& Lilien, L. T. (2014). Extending lifetime of wireless sensor networks by management of spare nodes. Procedia Computer Science, 34, 493-498.

[15] Bulut, E., \& Korpeoglu, I.(2011). Sleep scheduling with expected common coverage in wireless sensor networks. Wireless Networks, 17(1), 19-40.

[16] Cheng, C.T., Tse, C. K., \& Lau, F. C. M. (2011). A Clustering Algorithm for Wireless Sensor Networks Based on Social Insect Colonies. IEEE Sensor Journal, 11(3), 711-721.

[17] Rao, P. C. S., Jana, P. K., \& Banka, H. (2017). A particle swarm optimization based energy efficient cluster head selection algorithm for wireless sensor networks. Wireless Networks, 23(7), 2005-2020.

[18] Smaragdakis, G., Matta, I., \& Bestavros, A., (2004). Sep: A stable election protocol for clustered heterogeneous wireless sensor networks. Boston University Computer Science Department.

[19] Qing L., Zhu Q., \& Wang M. (2006). Design of a distributed energyefficient clustering algorithm for heterogeneous wireless sensor networks. Computer communications, 29(12), 2230-2237. 